\title{
Antidepressants: placebos with side effects?
}

\author{
The Emperor's New Drugs: Exploding the \\ Antidepressant Myth \\ Irving Kirsch \\ Basic Books; 2010.
}

I rving Kirsch, a professor of psychology at the University of Hull in the United Kingdom, has devoted much of his career to determining whether antidepressants are any more effective than placebos. His new book, The Emperor's New Drugs: Exploding the Antidepressant Myth, is a both a memoir of his controversial career and a review of the many studies about antidepressants and placebos, conducted both by himself and by others.

In his preface, Kirsch says that when he started writing the book, he was skeptical of the effectiveness of antidepressants, but was still willing to concede that they likely had some effectiveness for a subset of people. But when he finished reviewing all the data, "I realized that the situation was even worse than I thought. The belief that antidepressants can cure depression chemically is simply wrong." This is an extreme and provocative statement, and though Kirsch does a credible job of defending it, in the end I found his argument unconvincing.

The essential data underlying his book are a series of meta-analyses done by Kirsch and other researchers over the past decade. Typically, these studies have analyzed data from many randomized controlled trials of antidepressants submitted by drug companies to the US Food and Drug Administration (FDA). The consistent finding has been that, on average, antidepressants yield only a two-point advantage over placebo on the Hamilton Depression Scale, which is the main outcome measure used in antidepressant trials. Thus far, Kirsch is on firm ground, as this finding has been replicated and is in little dispute.

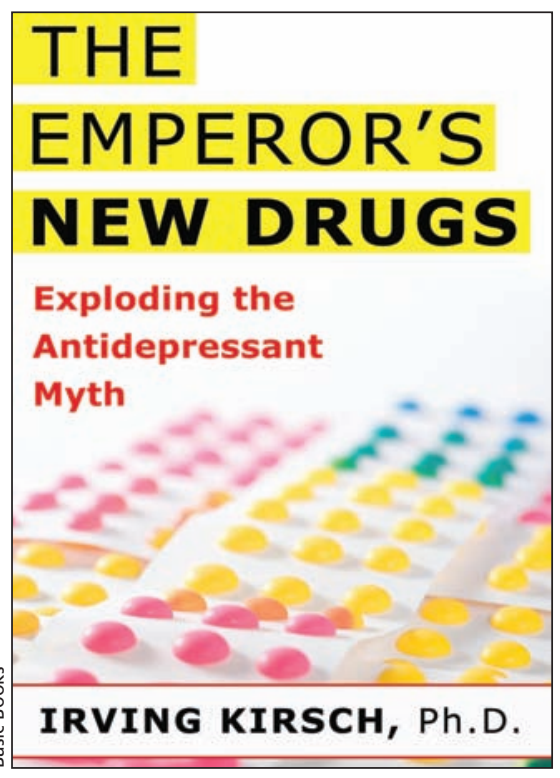

The controversy swirls around how we should interpret this data. Kirsch believes that this two-point gap, while statistically significant, is not clinically significant. He accurately points out that a two-point difference on the Hamilton scale "can be obtained by no longer waking during the night, or by no longer waking early in the morning, or by being less fidgety during the interview, or by eating better." These appear, indeed, to be clinically rather trivial improvements. But Kirsch drives several more nails into the antidepressant coffin by arguing that even this tiny two-point difference may be an artifact of the "breaking of the blind," which refers to patients realizing they were assigned to the active drug because they notice side effects. Thus, any apparent advantage of antidepressants over placebo may be simply an "augmented" placebo effect. The inescapable conclusion, according to Kirsch, is that antidepressants are simply placebos with side effects.

While Kirsch's reasoning is often impressive, he ignores at least two issues that render his conclusions suspect. First, antidepressants are robustly superior to placebo (both statistically and clinically) for a range of other psychiatric disorders which overlap in symptomology with depression, including panic disorder, generalized anxiety disorder, social anxiety disorder, obsessive compulsive disorder, and bulimia nervosa. While randomized controlled trials exclude patients with such comorbidities, in the real world most depressed patients also have anxiety disorders. Such patients probably truly do respond to antidepressants, even if this response is via the "back door" of anxiety disorders.

A second glaring issue ignored by Kirsch is a logical extension of his argument that antidepressants are placebos with side effects. If this is true, then it follows that any drug ever tested for depression should show at least a tiny improvement over actual placebo pills. But, in fact, this is not the case. For example, both antiepileptics and benzodiazepines have been evaluated in placebocontrolled trials for depression and most of the results have been negative. According to Kirsch's argument, since both of these classes of drugs have side effects, one would expect them to be as effective as any "antidepressant"- but they are not.

Notwithstanding these flaws, I still found the book an interesting read, particularly the last three chapters which focus on research attempting to define the mysterious placebo effect. I believe it is an important book, with the reservation that Kirsch's selective use of data gives him the appearance of an anti-antidepressant partisan, detracting from his overall persuasiveness.

\section{Daniel Carlat MD}

Associate clinical professor of psychiatry

Tufts University School of Medicine Newburyport, USA

Daniel Carlat is the publisher and editor of The Carlat Psychiatry Report. 